\title{
Dabco as an Inexpensive and Highly Efficient Ligand for Palladium-Catalyzed Suzuki-Miyaura Cross-Coupling Reaction
}

Jin-Heng $\mathrm{Li}^{*}$ and Wen-Jie Liu

Key Laboratory of Chemical Biology \& Traditional Chinese Medicine Research, College of Chemistry and Chemical Engineering, Hunan Normal University, Changsha 410081, China.

E-mail address:jhli@,mail.hunnu.edu.cn

\section{Supporting Information}

\section{List of Contents}
(A) General
(B) Materials
(C) Typical experimental procedures
(D) Analytical data for 3
(E) References
(F) NMR spectra 
(A) General

${ }^{1} \mathrm{H}$ and ${ }^{13} \mathrm{C}$ NMR spectra were recorded on an INOVA-400 (Varian) spectrometer.

(B) Materials

All reagents were directly used as obtained commercially. All products are known. ${ }^{1-6}$

(C) Typical experimental procedures

(1) Typical experimental procedure for the palladium-catalyzed Suzuki-Miyaura cross-coupling reaction (Tables 1 and 2)

A mixture of aryl halide $1(0.5 \mathrm{mmol})$, arylboronic acid $2(0.7 \mathrm{mmol}), \mathrm{Pd}(\mathrm{OAc})_{2}(3 \mathrm{~mol} \%)$, Dabco (6 mol\%), $\mathrm{K}_{2} \mathrm{CO}_{3}$ (3 equiv), and acetone $\left(5 \mathrm{~mL}\right.$ ) was added to a sealed tube, and stirred at $110{ }^{\circ} \mathrm{C}$ for desired time which monitored by TLC. After usual workup, the residue was purified by flash column chromatography to afford $\mathbf{3}$ (hexane/ethyl acetate).

Caution: Be careful because the reaction proceeded at higher boiling point with higher pressure

(2) Typical experimental procedure for $0.0001 \mathrm{~mol} \%$ of Pd and $0.0002 \mathrm{~mol} \%$ of Dabco-catalyzed coupling of 1-iodo-4-nitro-benzene (1a) with phenylboronic acid

\section{(2a) (Entry 4 in Table 3)}

Firstly, $\mathrm{Pd}(\mathrm{OAc})_{2}(4.5 \mathrm{mg}, 0.02 \mathrm{mmol})$ was dissolved in $200 \mathrm{~mL}$ of acetone, and Dabco (4.5 mg, $0.04 \mathrm{mmol})$ was also dissolved in another $200 \mathrm{~mL}$ of acetone. Then $50 \mu \mathrm{L}$ of $\mathrm{Pd}(\mathrm{OAc})_{2}$ acetone solution and $50 \mu \mathrm{L}$ of Dabco acetone solution were added to a mixture of 1-iodo-4-nitro-benzene 1a (5 mmol), phenylboronic acid $2 \mathbf{a}(7 \mathrm{mmol})$, and $\mathrm{K}_{2} \mathrm{CO}_{3}$ (3 equiv), and acetone (about $50 \mathrm{~mL}$ ) in a sealed tube, respectively (by syringe). The mixture was stirred at $110{ }^{\circ} \mathrm{C}$ for $48 \mathrm{~h}$, which monitored by TLC. After usual workup, the residue was purified by flash column chromatography (hexane) to afford $90 \%$ yield of 3a (about 89 mg, TONs: 900 000). 
(3) Typical experimental procedure for $0.0001 \mathrm{~mol} \%$ of $\mathrm{Pd}$ and $0.0002 \mathrm{~mol} \%$ of Dabco-catalyzed coupling of iodobenzene (1d) with $p$-chlorophenylboronic acid (2d) (Entry 5 in Table 3)

Firstly, $\mathrm{Pd}(\mathrm{OAc})_{2}(4.5 \mathrm{mg}, 0.02 \mathrm{mmol})$ was dissolved in $200 \mathrm{~mL}$ of acetone, and Dabco (4.5 mg, $0.04 \mathrm{mmol})$ was also dissolved in another $200 \mathrm{~mL}$ of acetone. Then $5 \mu \mathrm{L}$ of $\mathrm{Pd}(\mathrm{OAc})_{2}$ acetone solution and $5 \mu \mathrm{L}$ of Dabco acetone solution were added to a mixture of iodobenzene $1 \mathbf{d}(0.5 \mathrm{mmol})$, p-chlorophenylboronic acid $\mathbf{2 d}(0.7 \mathrm{mmol})$, and $\mathrm{K}_{2} \mathrm{CO}_{3}$ (3 equiv), and acetone (about $5 \mathrm{~mL}$ ) in a sealed tube, respectively (by syringe). The mixture was stirred at $110^{\circ} \mathrm{C}$ for $14 \mathrm{~h}$, which monitored by TLC. After usual workup, the residue was purified by flash column chromatography (hexane) to afford $95 \%$ yield of $\mathbf{3 f}$ (about $89 \mathrm{mg}$, TONs: 950 000).

(4) Typical experimental procedure for $0.001 \mathrm{~mol} \%$ of Pd and $0.002 \mathrm{~mol} \%$ of Dabco-catalyzed coupling of 1-bromo-4-methoxybenzene (1h) with phenylboronic acid (2a) (Entry 14 in Table 3)

Firstly, $\mathrm{Pd}(\mathrm{OAc})_{2}(4.5 \mathrm{mg}, 0.02 \mathrm{mmol})$ was dissolved in $200 \mathrm{~mL}$ of acetone, and Dabco (4.5 mg, $0.04 \mathrm{mmol})$ was also dissolved in another $200 \mathrm{~mL}$ of acetone. Then $50 \mu \mathrm{L}$ of $\mathrm{Pd}(\mathrm{OAc})_{2}$ acetone solution and $50 \mu \mathrm{L}$ of Dabco acetone solution were added to a mixture of 1-bromo-4-methoxybenzene 1h $(0.5 \mathrm{mmol})$, phenylboronic acid $\mathbf{2 a}(0.7 \mathrm{mmol})$, and $\mathrm{K}_{2} \mathrm{CO}_{3}$ (3 equiv), and acetone (about $5 \mathrm{~mL}$ ) in a sealed tube, respectively (by syringe). The mixture was stirred at $110{ }^{\circ} \mathrm{C}$ for $48 \mathrm{~h}$, which monitored by TLC. After usual workup, the residue was purified by flash column chromatography (hexane/ethyl acetate) to afford 9\% yield of $\mathbf{3 g}$ (about $8 \mathrm{mg}$, TONs: 9000 ), and $>85 \%$ of 1-bromo-4-methoxybenzene 1h was recovered. 


\section{(D) Analytical data for 3}

\section{4-Nitro-biphenyl (3a) ${ }^{1-3}$}

Pale yellow solid; ${ }^{1} \mathrm{H}$ NMR (400 MHz, $\left.\mathrm{CDCl}_{3}\right): \delta 8.31(\mathrm{~d}, J=8.4 \mathrm{~Hz}, 2 \mathrm{H}), 7.75(\mathrm{~d}, J=8.8 \mathrm{~Hz}$, 2H), $7.64(\mathrm{~d}, J=7.2 \mathrm{~Hz}, 2 \mathrm{H}), 7.53-7.46(\mathrm{~m}, 3 \mathrm{H}) ;{ }^{13} \mathrm{C} \mathrm{NMR}\left(100 \mathrm{MHz}, \mathrm{CDCl}_{3}\right) \delta: 147.6,147.1$, $138.8,129.2,128.91,127.8,127.4,124.1$.

\section{3-Nitro-biphenyl $(3 b)^{2}$}

White solid; ${ }^{1} \mathrm{H}$ NMR (400 MHz, $\left.\mathrm{CDCl}_{3}\right): \delta 8.47(\mathrm{~s}, 1 \mathrm{H}), 8.21(\mathrm{~d}, J=8 \mathrm{~Hz}, 1 \mathrm{H}), 7.91(\mathrm{~d}, J=8$ $\mathrm{Hz}, 1 \mathrm{H}), 7.65-7.60(\mathrm{~m}, 3 \mathrm{H}), 7.51(\mathrm{t}, J=8 \mathrm{~Hz}, 2 \mathrm{H}), 7.44(\mathrm{t}, J=7.8 \mathrm{~Hz}, 1 \mathrm{H}) ;{ }^{13} \mathrm{C} \mathrm{NMR}(100 \mathrm{MHz}$, $\left.\mathrm{CDCl}_{3}\right): \delta 137.5,129.0,128.9,127.9,126.8$.

$\mathrm{NO}_{2}$

\section{2-Nitro-biphenyl (3c) $)^{1}$}

Pale yellow oil; ${ }^{1} \mathrm{H}$ NMR (400 MHz, $\left.\mathrm{CDCl}_{3}\right): \delta 7.86(\mathrm{~d}, J=8 \mathrm{~Hz}, 1 \mathrm{H}), 7.62(\mathrm{t}, J=7.2 \mathrm{~Hz}, 1 \mathrm{H})$, $7.51-7.40(\mathrm{~m}, 5 \mathrm{H}), 7.34-7.31(\mathrm{~m}, 2 \mathrm{H}) ;{ }^{13} \mathrm{C}$ NMR $\left(100 \mathrm{MHz}, \mathrm{CDCl}_{3}\right): \delta 163.7,140.3,137.3$, $128.8,128.7,128.6,127.3,127.0,115.7,115.5$.

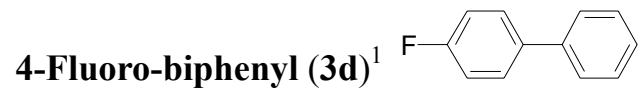

White solid; ${ }^{1} \mathrm{H}$ NMR (400 MHz), $\left.\mathrm{CDCl}_{3}\right): \delta 7.57-7.53(\mathrm{~m}, 4 \mathrm{H}), 7.44(\mathrm{t}, J=7.6 \mathrm{~Hz}, 2 \mathrm{H}), 7.35$ $(\mathrm{t}, J=7.2 \mathrm{~Hz}, 1 \mathrm{H}), 7.13(\mathrm{t}, J=8.8 \mathrm{~Hz}, 2 \mathrm{H}) ;{ }^{13} \mathrm{C} \mathrm{NMR}\left(100 \mathrm{MHz}, \mathrm{CDCl}_{3}\right): \delta 162.5(\mathrm{~d}, J=244.9$ $\mathrm{Hz}), 161.2,140.2,137.3,128.8,127.2,115.6(\mathrm{~d}, J=21.8 \mathrm{~Hz})$. 
4-Trifluoromethyl-biphenyl $(3 e)^{3} \mathrm{CF}_{3}$

White solid; ${ }^{1} \mathrm{H}$ NMR (400 MHz, $\left.\mathrm{CDCl}_{3}\right): \delta 7.69(\mathrm{~s}, 4 \mathrm{H}), 7.60(\mathrm{~d}, J=8 \mathrm{~Hz}, 2 \mathrm{H}), 7.48(\mathrm{t}, J=7.2$ $\mathrm{Hz}, 2 \mathrm{H}), 7.40(\mathrm{t}, J=7.2 \mathrm{~Hz}, 1 \mathrm{H}) ;{ }^{13} \mathrm{C} \mathrm{NMR}\left(100 \mathrm{MHz}, \mathrm{CDCl}_{3}\right): \delta 144.7,139.8,129.0,128.2$, $127.6,127.4,127.3,125.9125 .7$.

4-Chloro-biphenyl (3f) $)^{1,2} \mathrm{Cl}$

White solid; ${ }^{1} \mathrm{H}$ NMR (400 MHz, $\left.\mathrm{CDCl}_{3}\right): \delta 7.57-7.49(\mathrm{~m}, 4 \mathrm{H}), 7.46-7.34(\mathrm{~m}, 5 \mathrm{H}) ;{ }^{13} \mathrm{C}$ NMR (100 MHz, $\left.\mathrm{CDCl}_{3}\right): \delta 140.0,139.6,133.3,129.0,128.9,128.4,127.6,127.0$.

4-Methoxy-biphenyl (3g) $)^{1,4} \mathrm{MeO}-$

White solid; ${ }^{1} \mathrm{H}$ NMR (400 MHz, $\left.\mathrm{CDCl}_{3}\right): \delta 7.55$ (t, $\left.J=8.8 \mathrm{~Hz}, 4 \mathrm{H}\right), 7.42(\mathrm{t}, J=8 \mathrm{~Hz}, 2 \mathrm{H})$, $7.31(\mathrm{t}, J=7.2,1 \mathrm{H}), 6.99(\mathrm{~d}, \mathrm{~J}=12,2 \mathrm{H}), 1.57(\mathrm{~s}, 3 \mathrm{H}) ;{ }^{13} \mathrm{C} \mathrm{NMR}\left(100 \mathrm{MHz}, \mathrm{CDCl}_{3}\right): \delta 159.1$, $140.7,133.7,128.7,128.1,126.7,126.6,114.2,55.3$.

1-Biphenyl-4-yl-ethanone $(3 \mathrm{~h})^{4}$ $-\mathrm{COCH}_{3}$

White solid; ${ }^{1} \mathrm{H}$ NMR (400 MHz, $\left.\mathrm{CDCl}_{3}\right): \delta 8.04(\mathrm{~d}, J=8.4 \mathrm{~Hz}, 2 \mathrm{H}), 7.69(\mathrm{~d}, J=8.4 \mathrm{~Hz}, 2 \mathrm{H})$, $7.64(\mathrm{~d}, J=7.6 \mathrm{~Hz}, 2 \mathrm{H}), 7.48(\mathrm{t}, J=7.6 \mathrm{~Hz}, 2 \mathrm{H}), 7.41(\mathrm{t}, J=7.2 \mathrm{~Hz}, 1 \mathrm{H}) ;{ }^{13} \mathrm{C} \mathrm{NMR}(100 \mathrm{MHz}$, $\left.\mathrm{CDCl}_{3}\right): \delta 197.8,145.8,139.9,135.8,128.9,128.9,128.2,127.3,127.2,26.7$.

1-(4'-Fluoro-biphenyl-4-yl)-ethanone $(3 \mathbf{i})^{5}$

White solid; ${ }^{1} \mathrm{H}$ NMR (400 MHz, $\left.\mathrm{CDCl}_{3}\right): \delta 8.03(\mathrm{~d}, J=8 \mathrm{~Hz}, 2 \mathrm{H}), 7.64(\mathrm{~d}, J=8 \mathrm{~Hz}, 2 \mathrm{H}), 7.60$ $-7.58(\mathrm{~m}, 2 \mathrm{H}), 7.17(\mathrm{t}, \mathrm{J}=8.4 \mathrm{~Hz}, 2 \mathrm{H}), 2.65(\mathrm{~s}, 3 \mathrm{H}) ;{ }^{13} \mathrm{C} \mathrm{NMR}\left(100 \mathrm{MHz}, \mathrm{CDCl}_{3}\right): \delta$ 197.7, 
$163.0(J=306.9 \mathrm{~Hz}), 144.7,135.9,135.8,129.0,128.9,127.1,116.0(\mathrm{~d}, J=21.1 \mathrm{~Hz}), 26.7$.

4, 4"'-Difluoro-[1,1';4',1"]terphenyl (3j) $)^{6}$

White solid; ${ }^{1} \mathrm{H}$ NMR $\left(400 \mathrm{MHz}, \mathrm{CDCl}_{3}\right): \delta 7.51-7.48(\mathrm{~m}, 6 \mathrm{H}), 7.14-7.10(\mathrm{~m}, 6 \mathrm{H}) ;{ }^{13} \mathrm{C}$ NMR $\left(100 \mathrm{MHz}, \mathrm{CDCl}_{3}\right): \delta 162.4(\mathrm{~d}, J=244.7 \mathrm{~Hz}), 136.4,131.9,128.6,128.5,115.7(\mathrm{~d}, J=21.8$ $\mathrm{Hz})$.

\section{(E) References}

(1) Beadle, J. B.; Korzeniowski, S. H.; Rosenberg, D. E.; Garcia-Slanga, B. J.; Gokel, G. W. J. Org.Chem. 1984, 49, 1594.

(2) Tao, B.; Boykin, D. W. Tetrahedron Lett. 2002, 43, 4955.

(3) Su, W.; Urgaonkar, S.; Verkade, J. G. Org. Lett. 2004, 6, 1421.

(4) Tang, Z.-Y.; Hu, Q.-S. J. Am. Chem. Soc. 2004, 126, 3058.

(5) Marie, F.; Dorothee, L.; Henri, D.; Maurice, S. Synthesis, 2001, 2320.

(6) Kamata, M.; Satoh, C.; Kim, H.-S.; Wataya, Y. Tetrahedron Lett. 2002, 43, 8313. 
(F) NMR spectra

4-Nitro-biphenyl (3a)
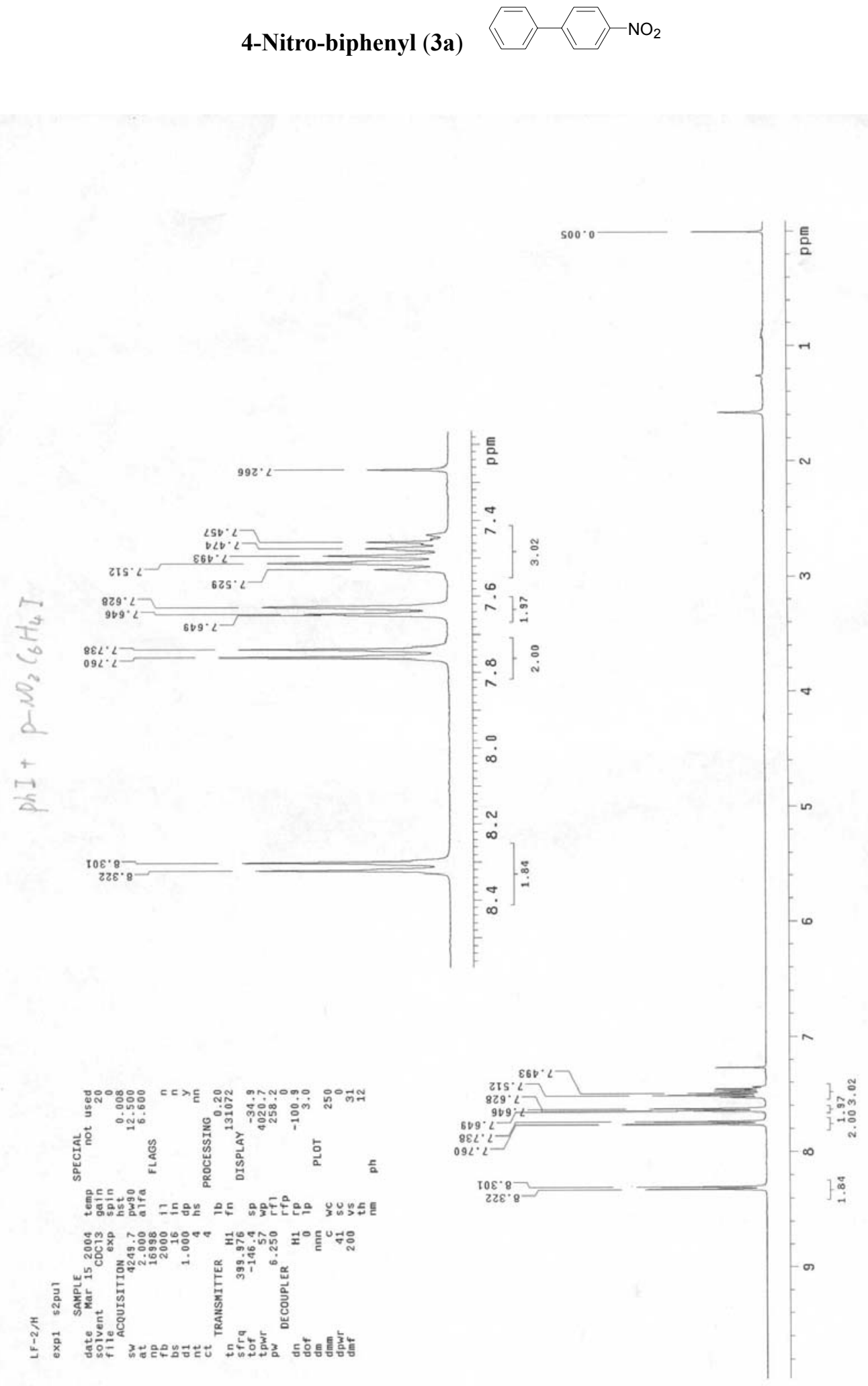

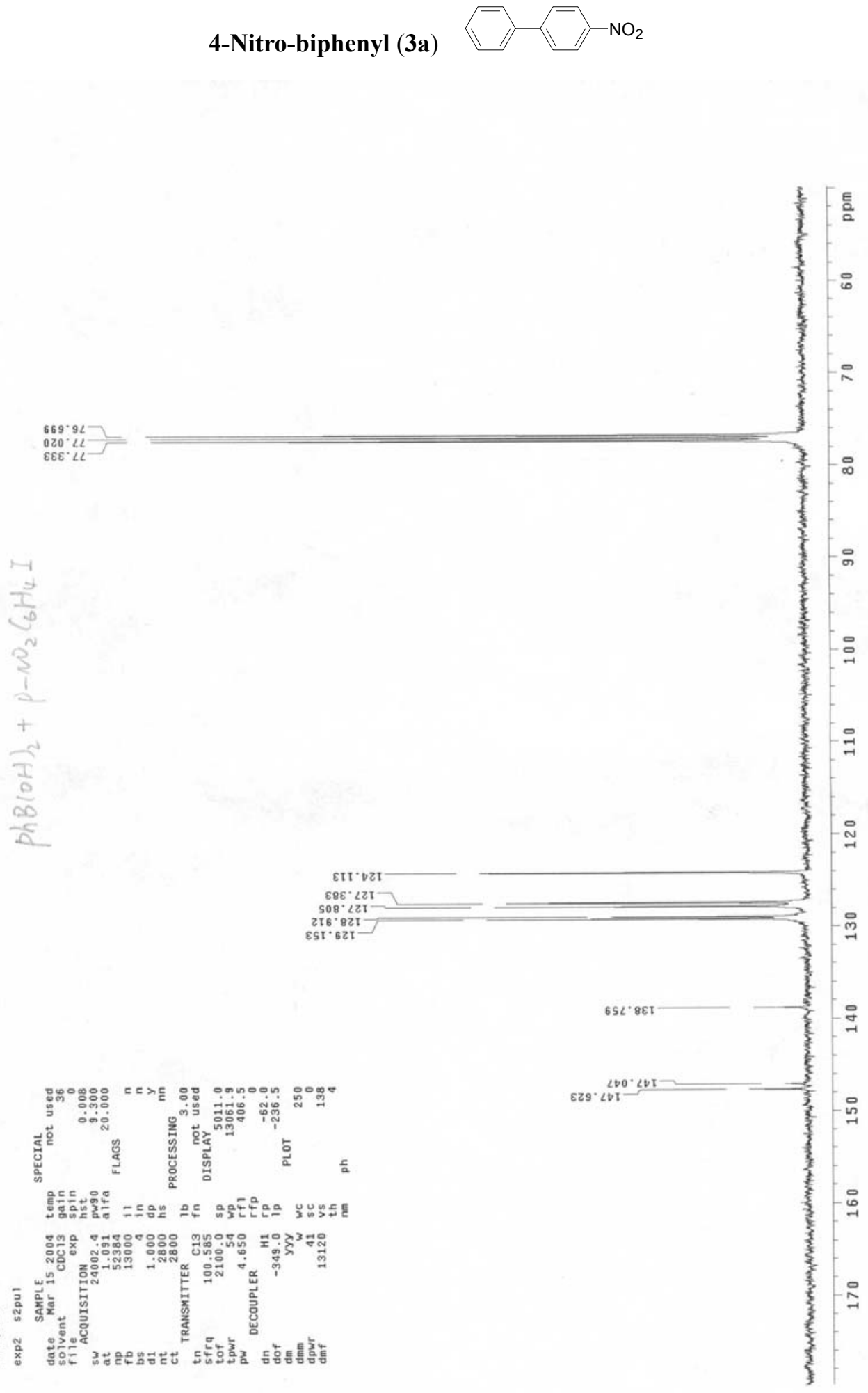


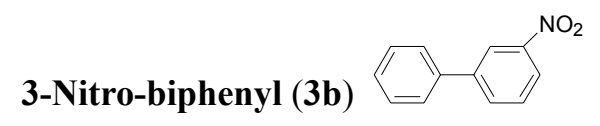

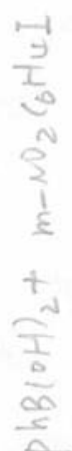

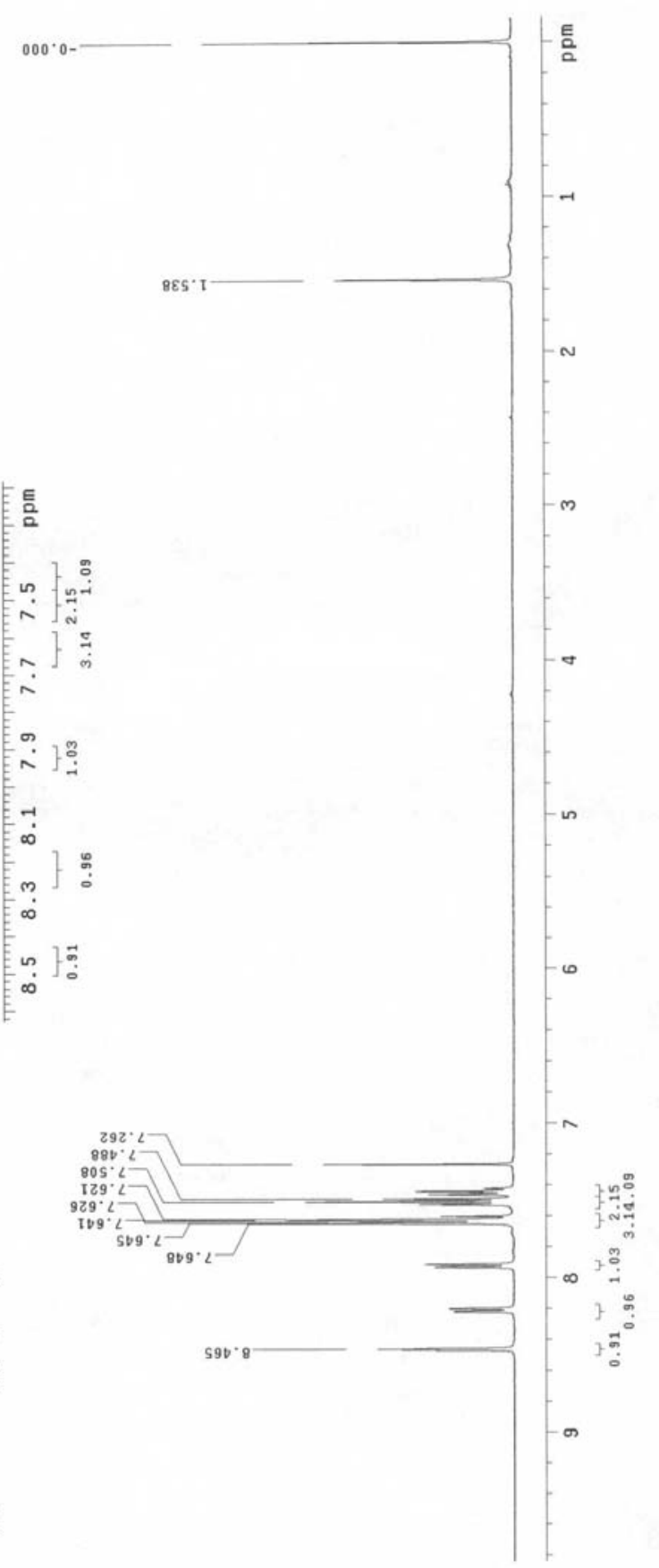




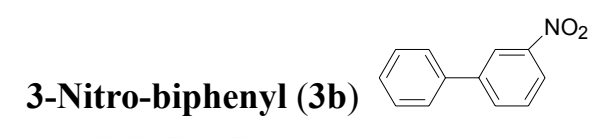

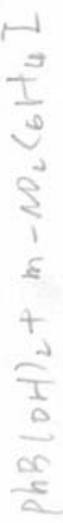

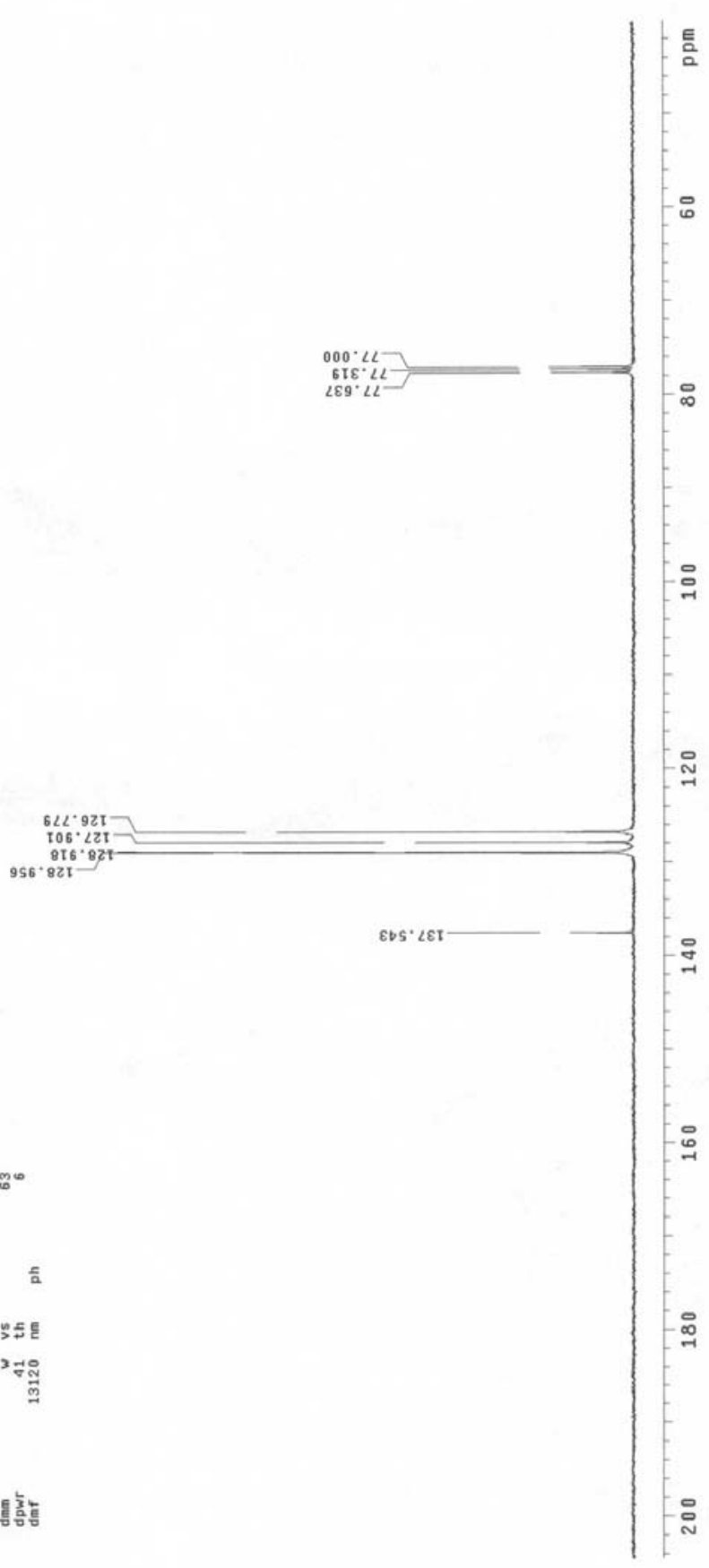

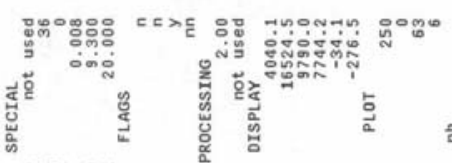

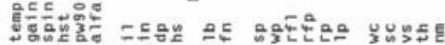

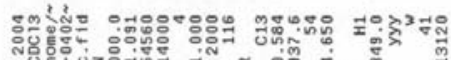

言密卷

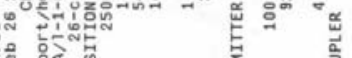

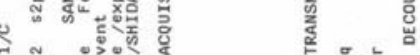

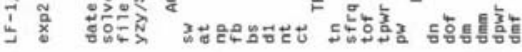


2-Nitro-biphenyl (3c)

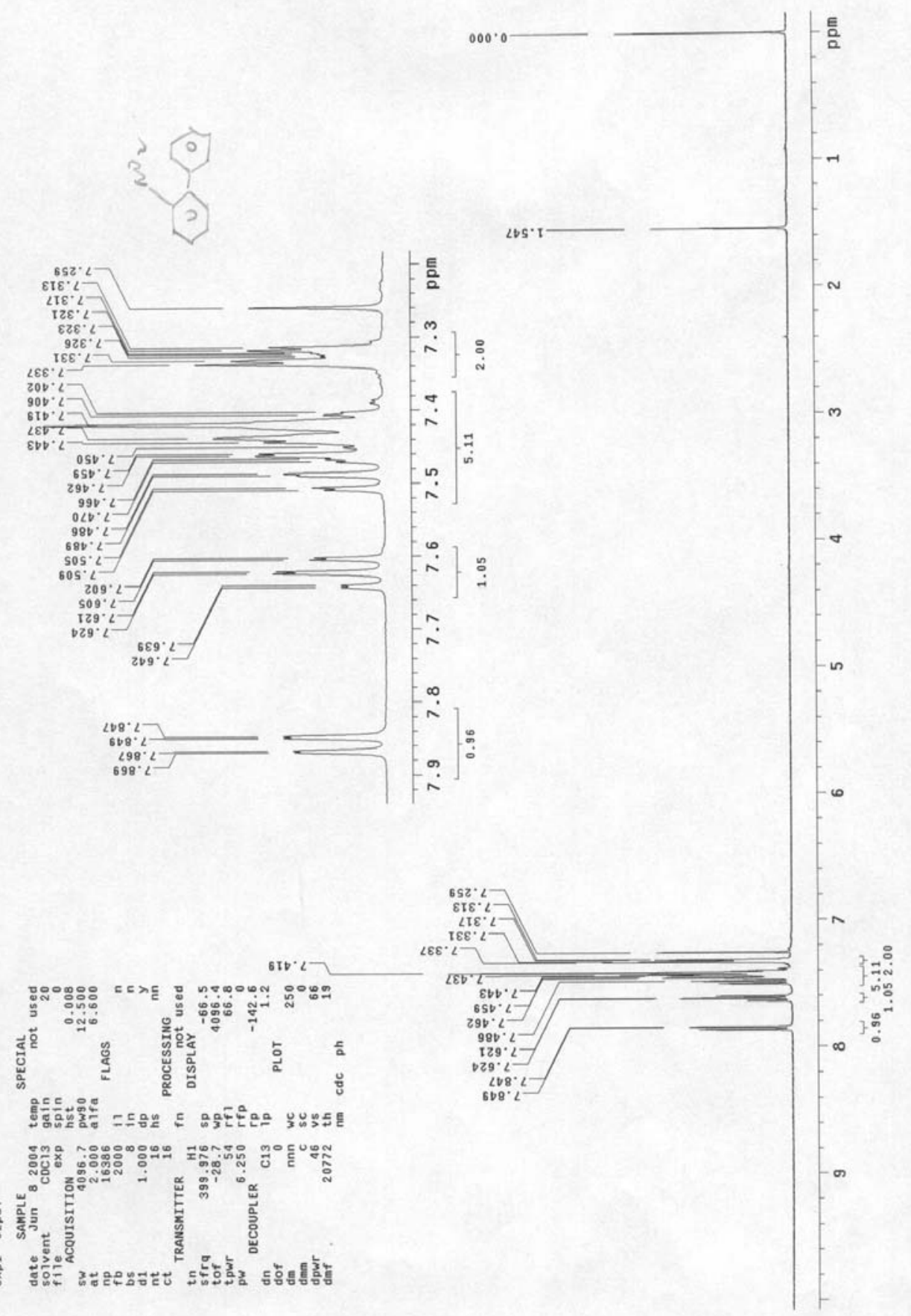


2-Nitro-biphenyl (3c)

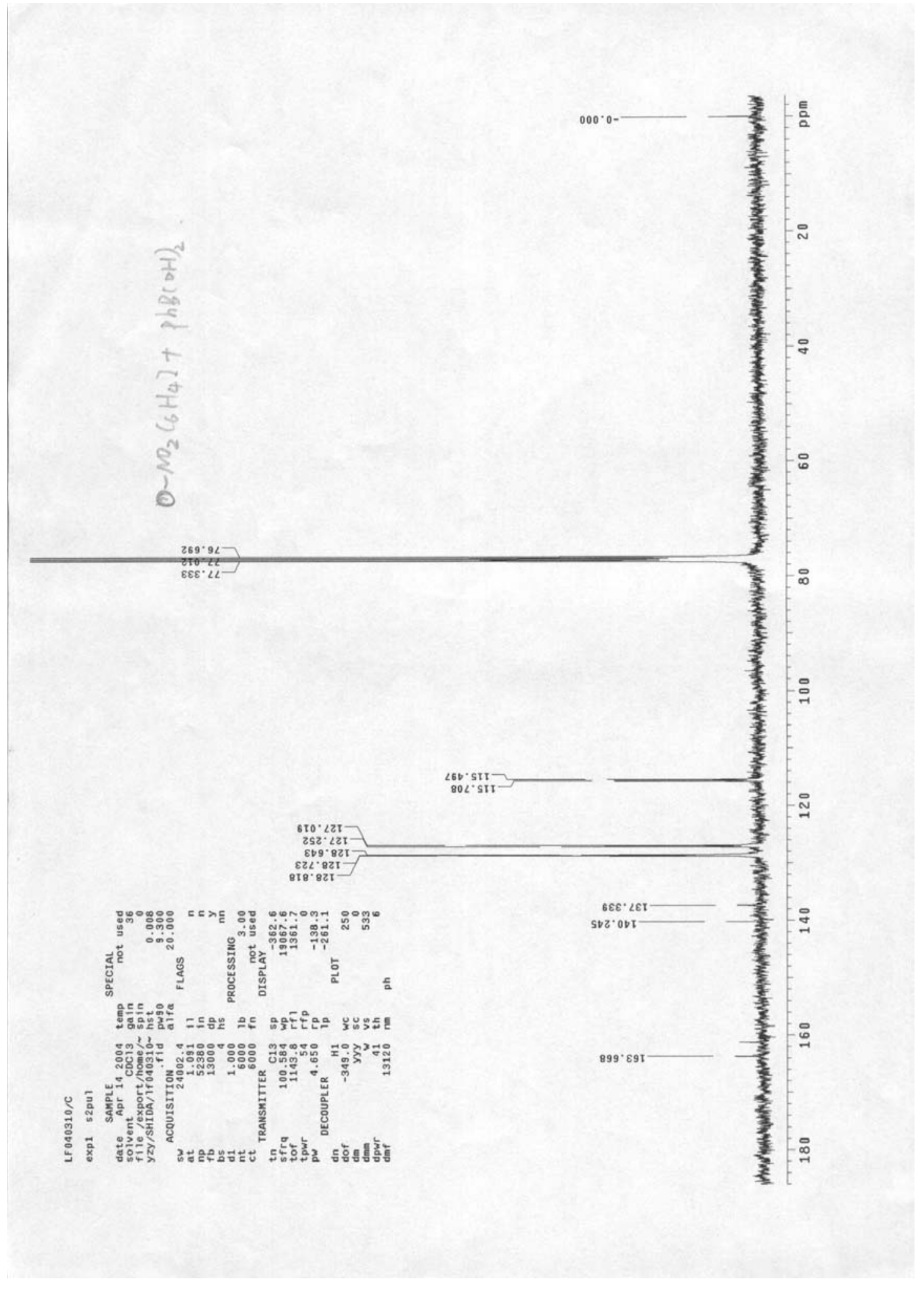


4-Fluoro-biphenyl (3d)

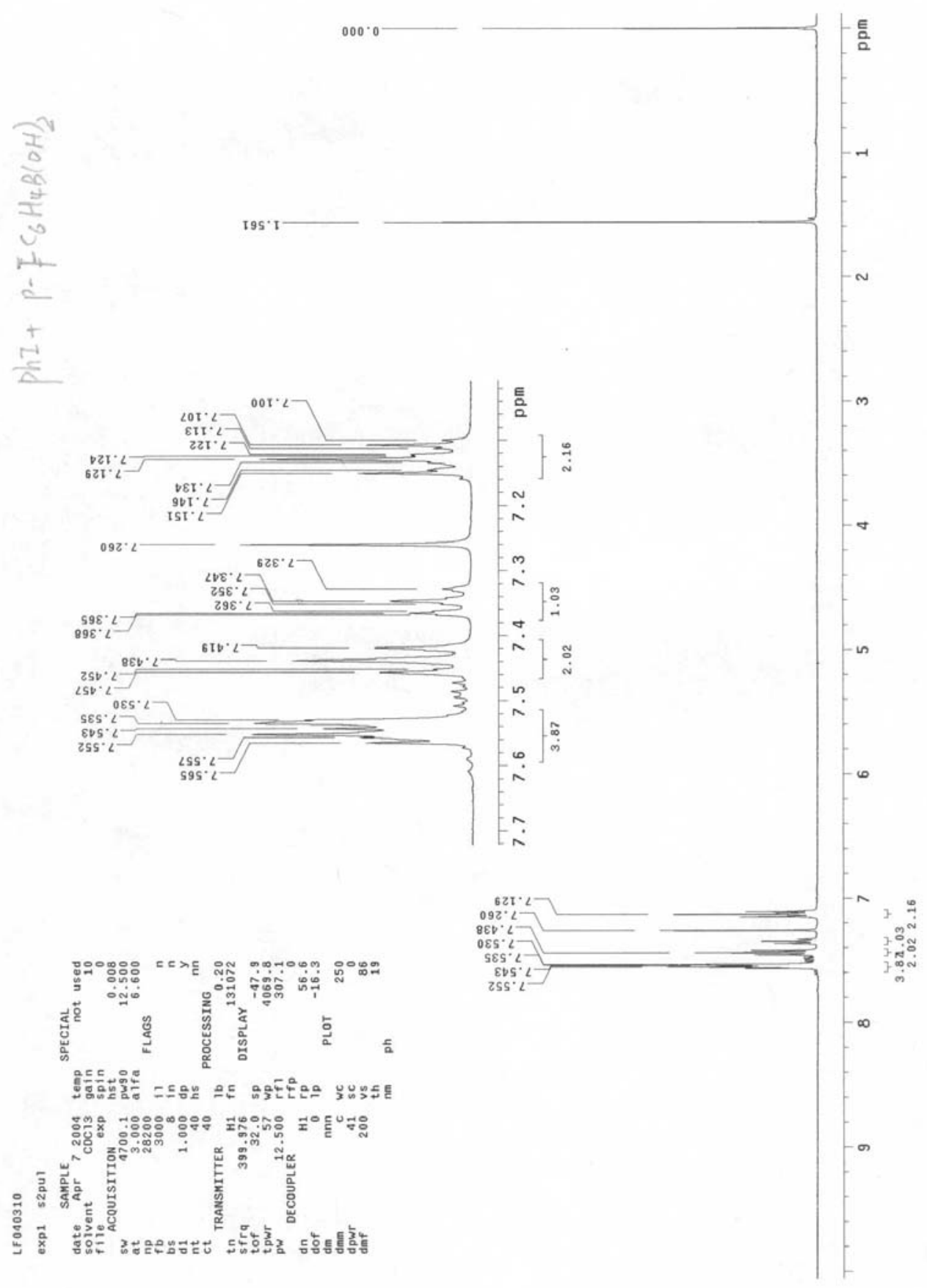


4-Fluoro-biphenyl (3d)

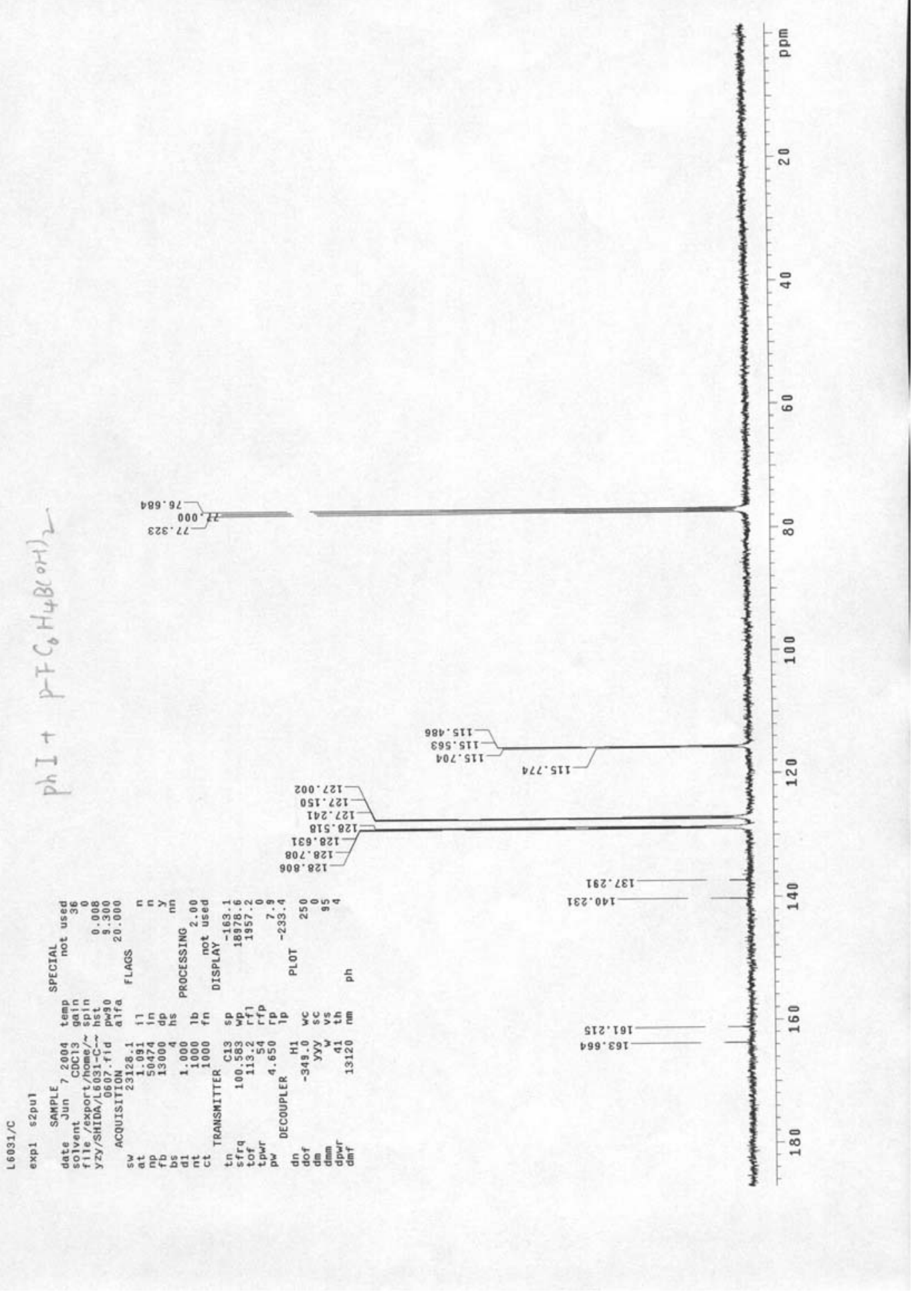


4-Trifluoromethyl-biphenyl (3e) $\mathrm{CF}_{3}$

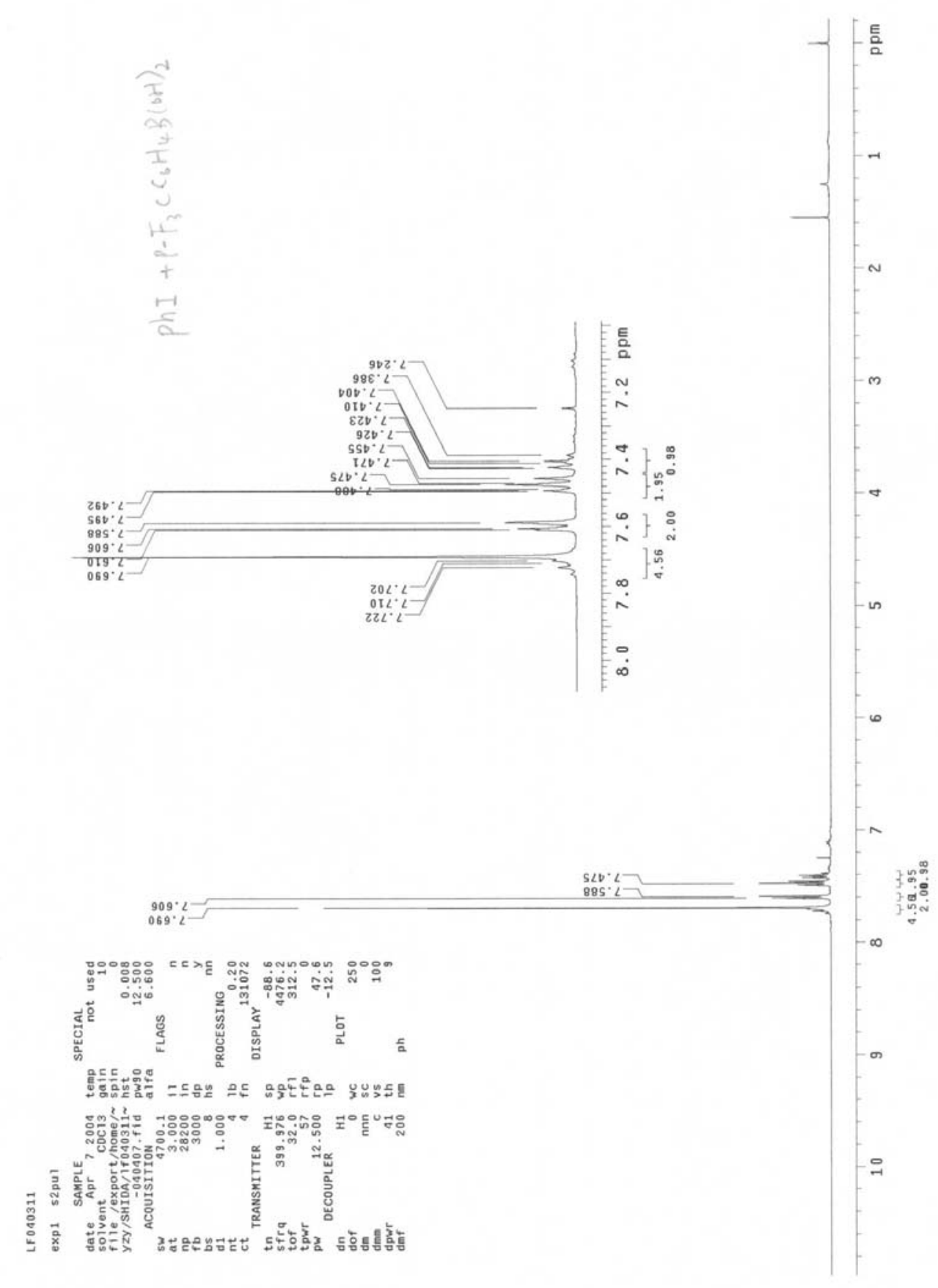


4-Trifluoromethyl-biphenyl (3e) ${ }^{\mathrm{CF}_{3}-}$

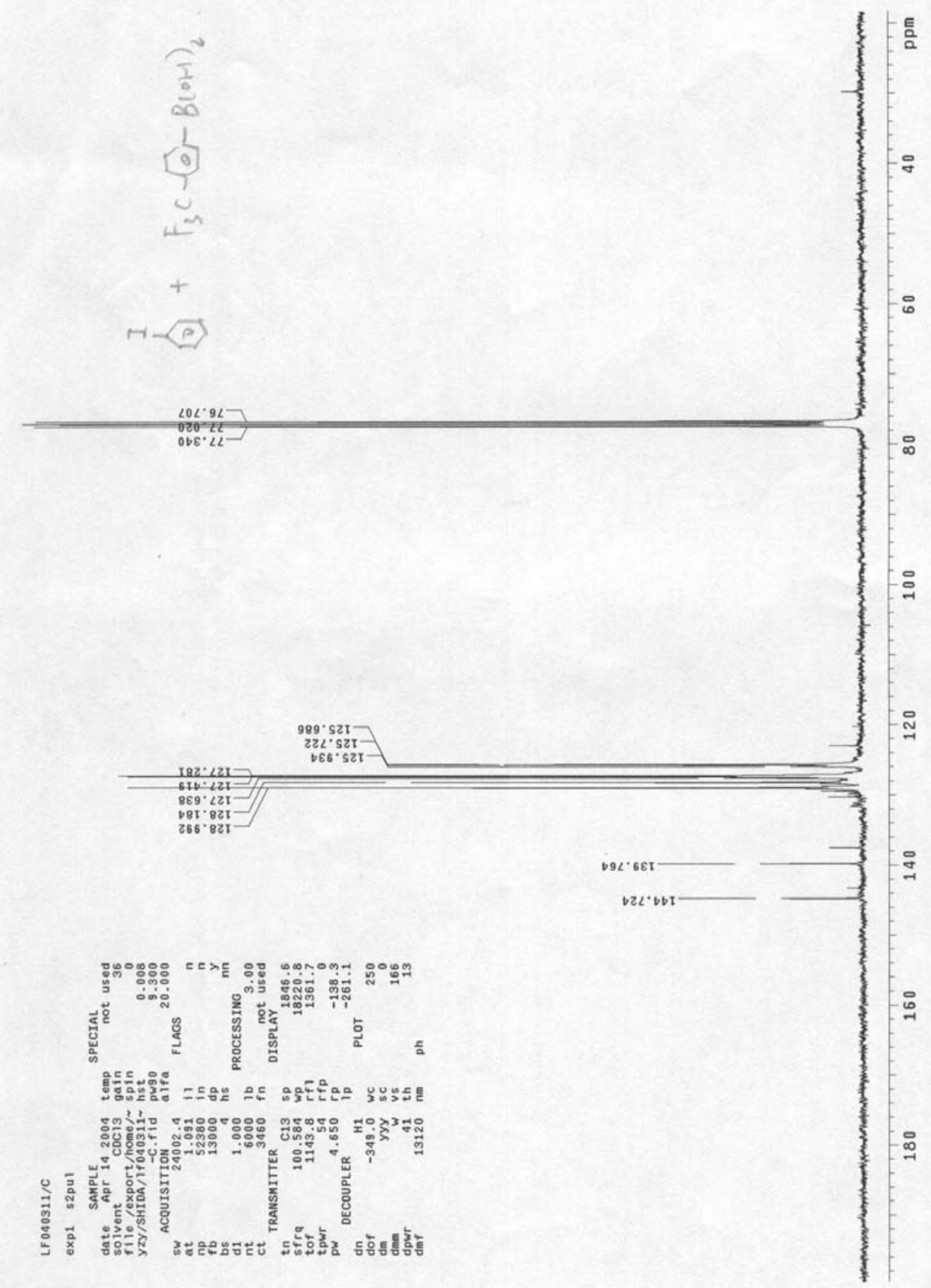


4-Chloro-biphenyl (3f)
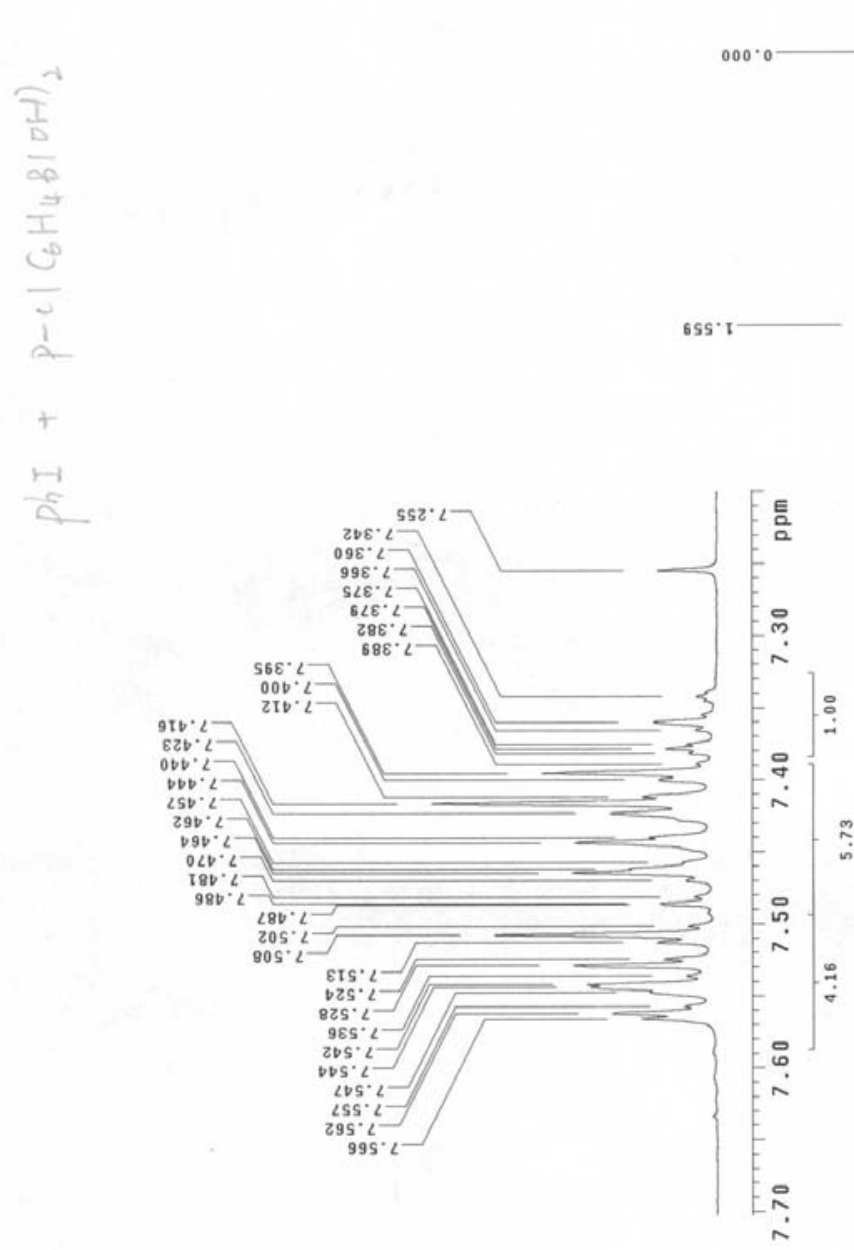

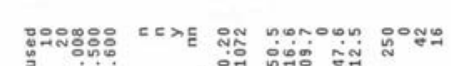

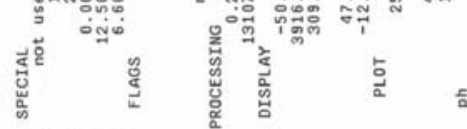

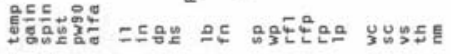

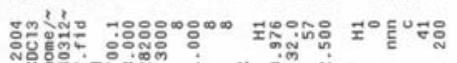

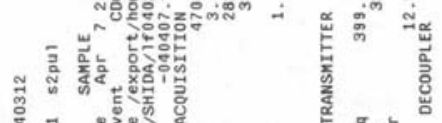

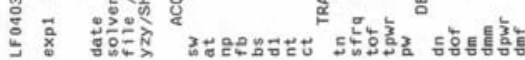

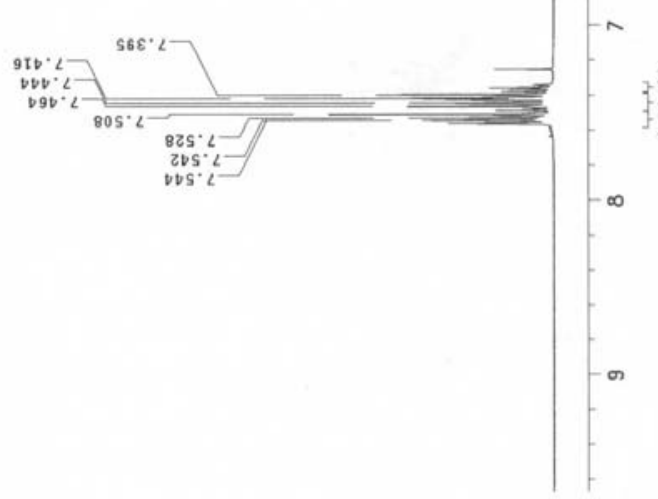


4-Chloro-biphenyl (3f)

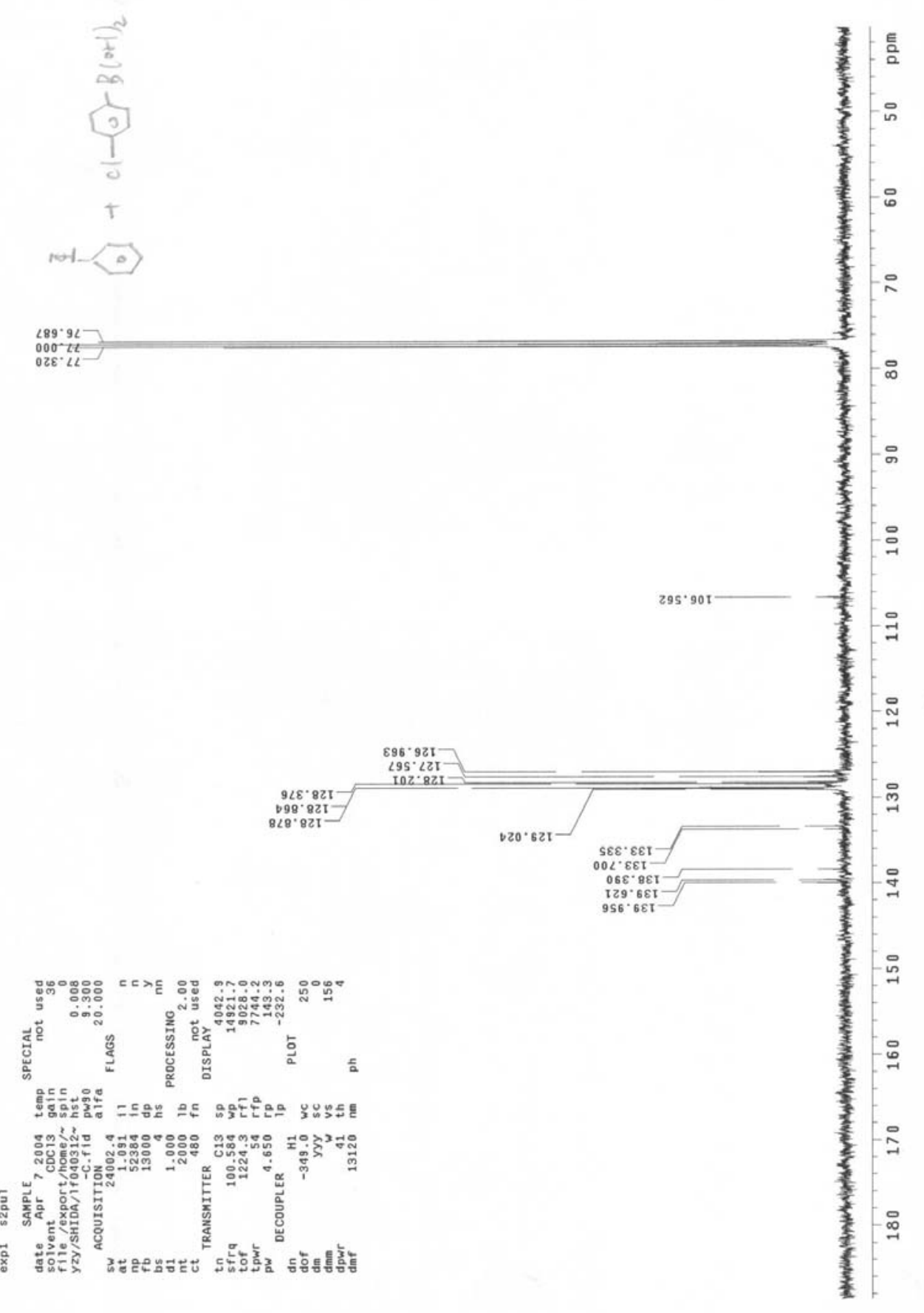


4-Methoxy-biphenyl (3g) $\mathrm{MeO}$

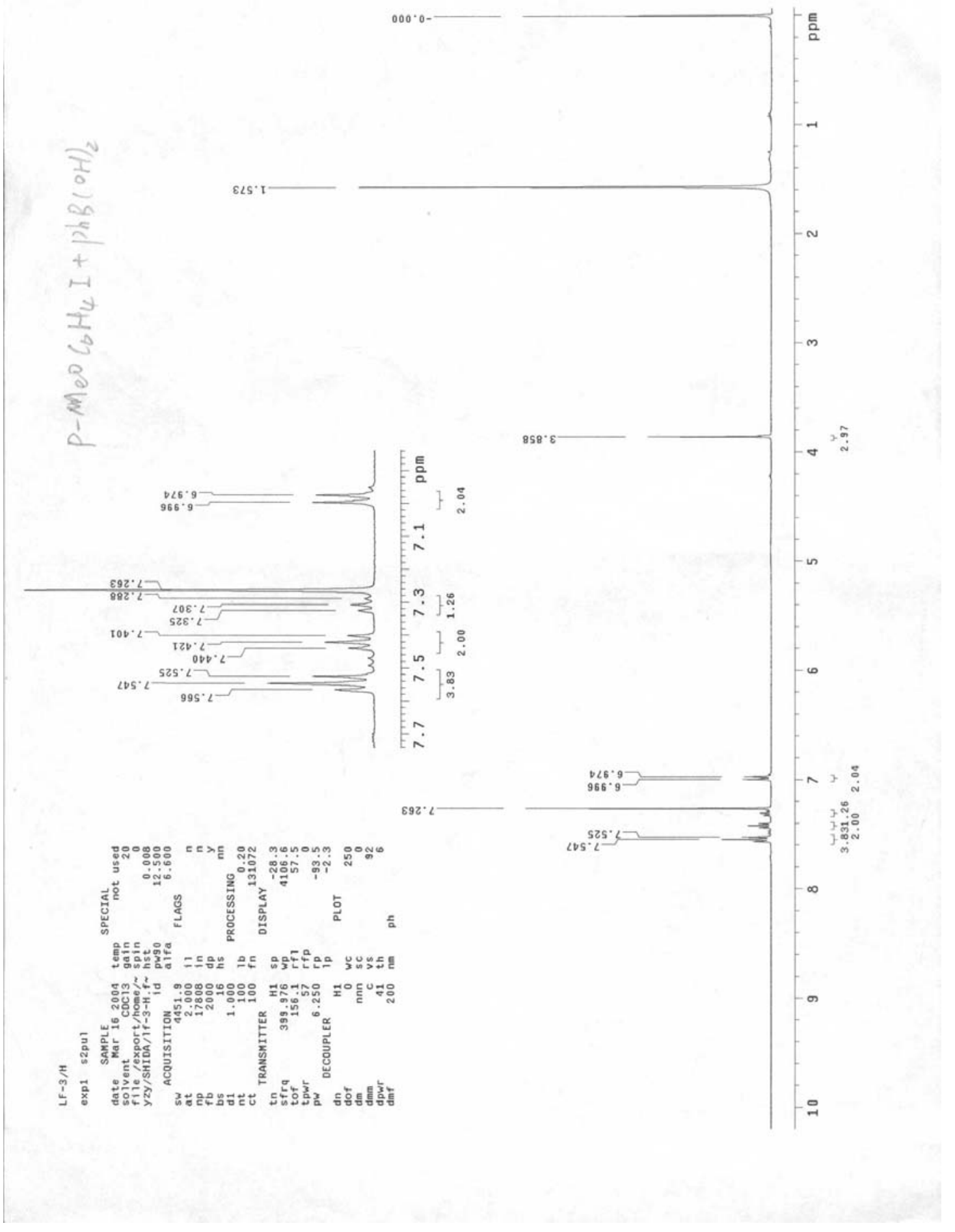


4-Methoxy-biphenyl (3g) MeO-
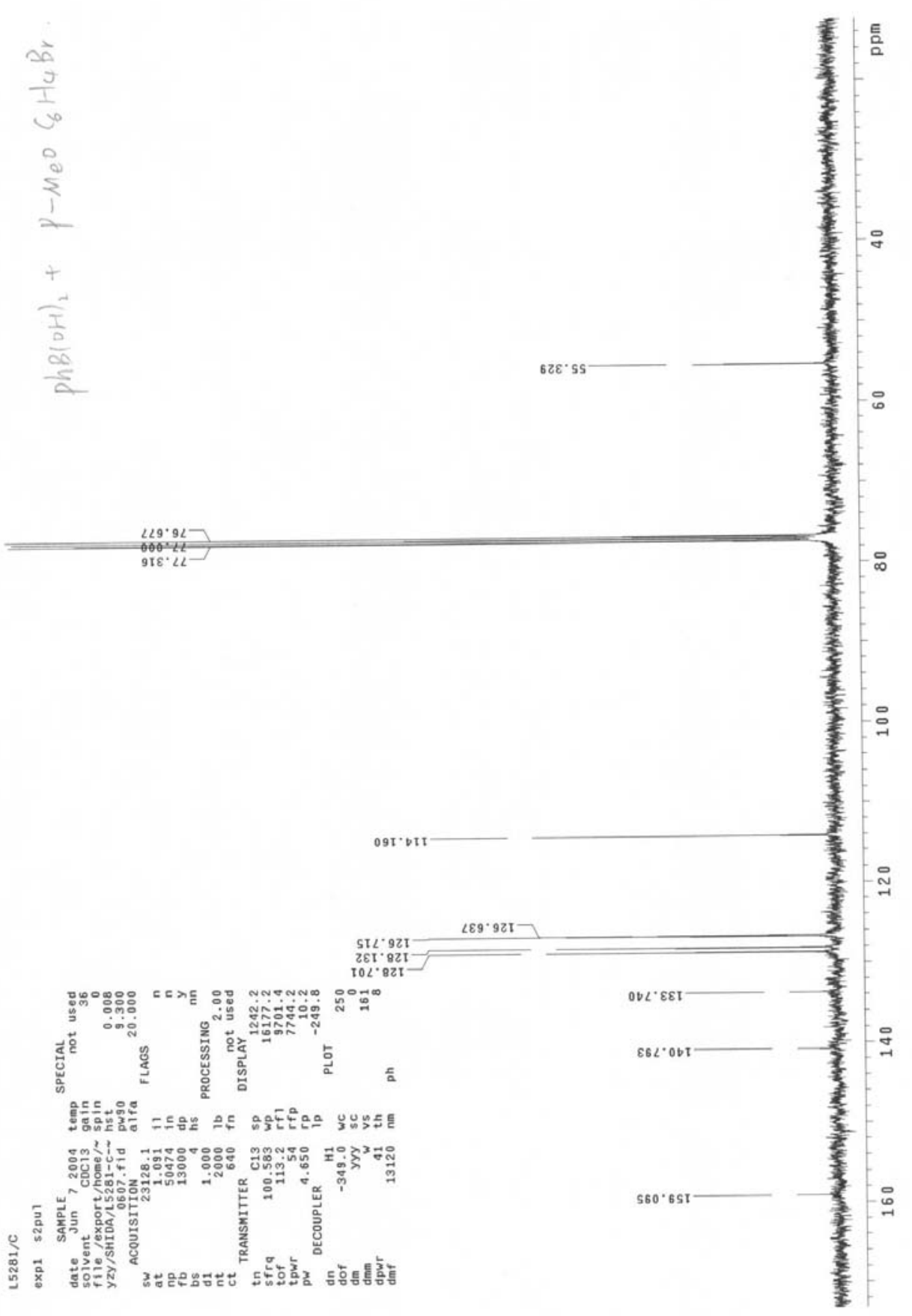
1-Biphenyl-4-yl-ethanone (3h)
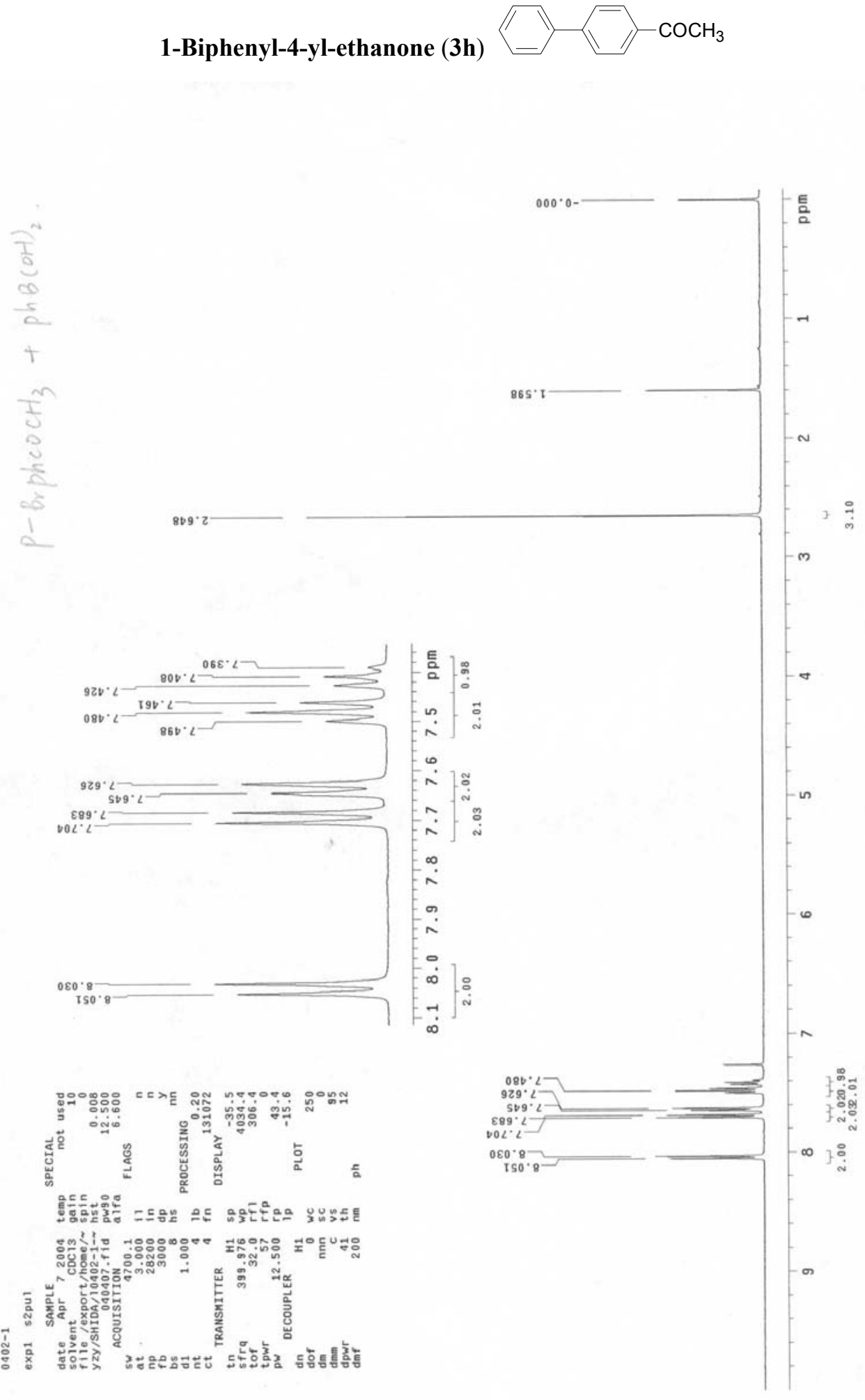
1-Biphenyl-4-yl-ethanone (3h)
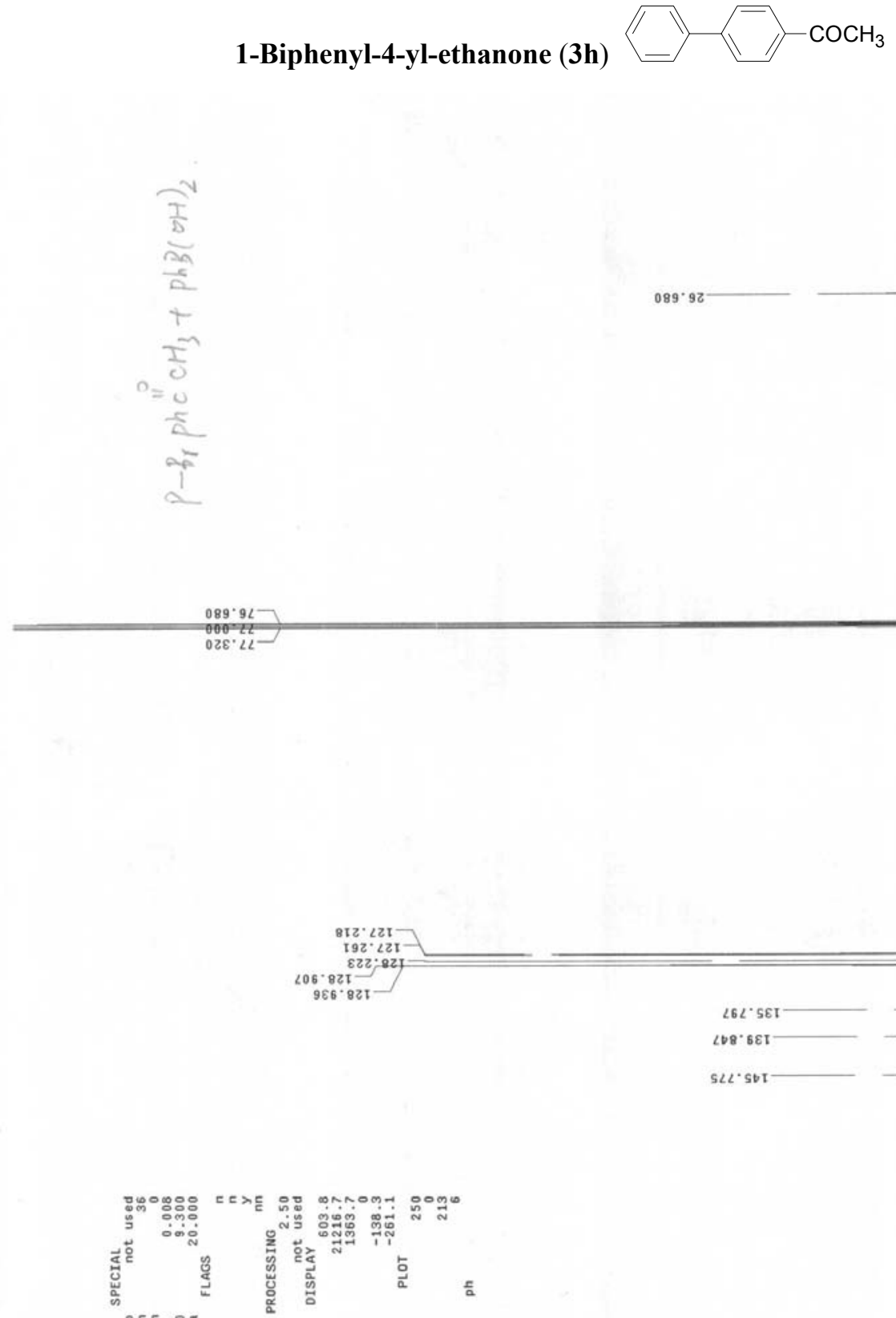

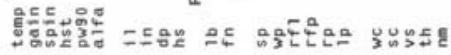
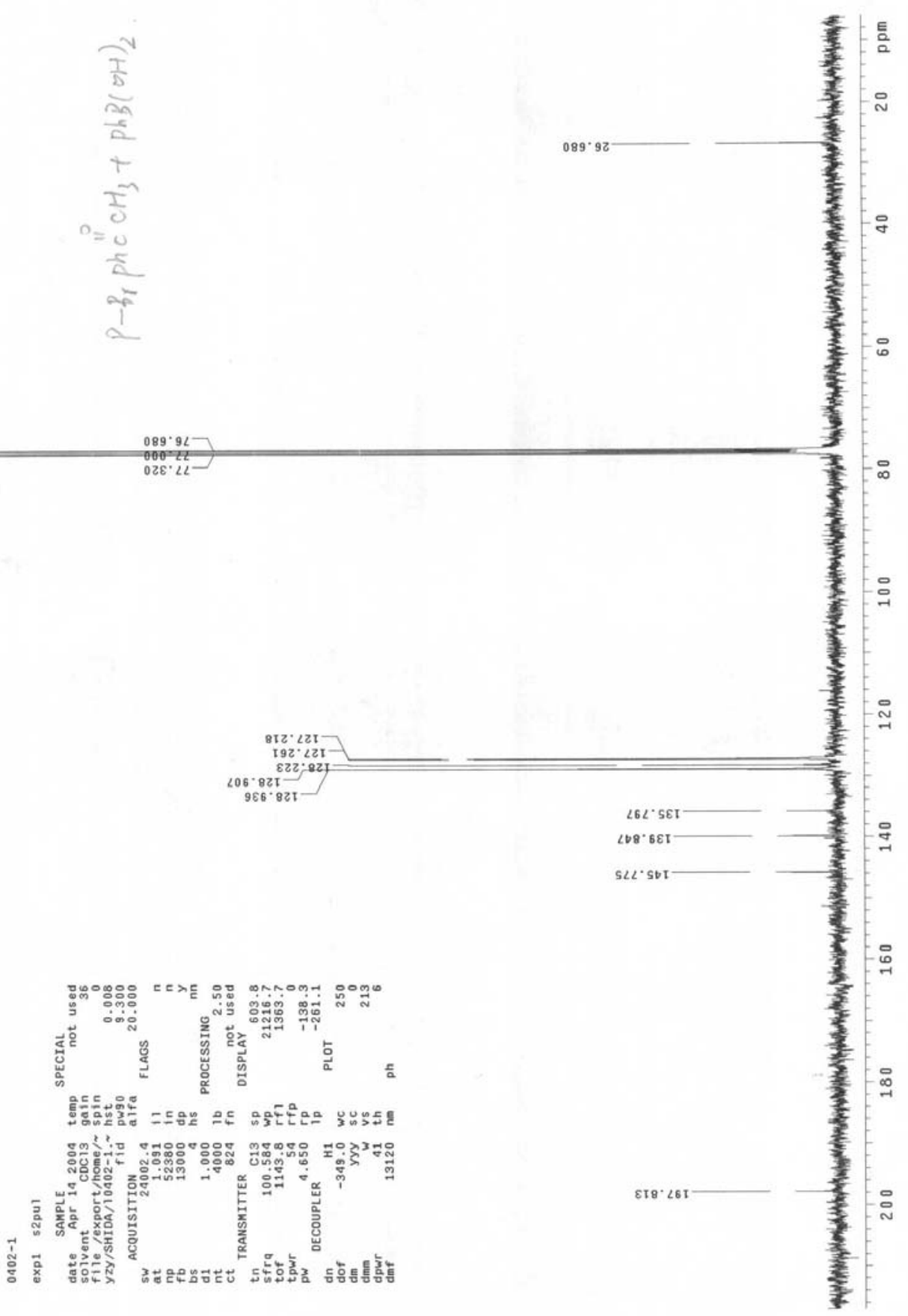
1-(4'-Fluoro-biphenyl-4-yl)-ethanone (3i)

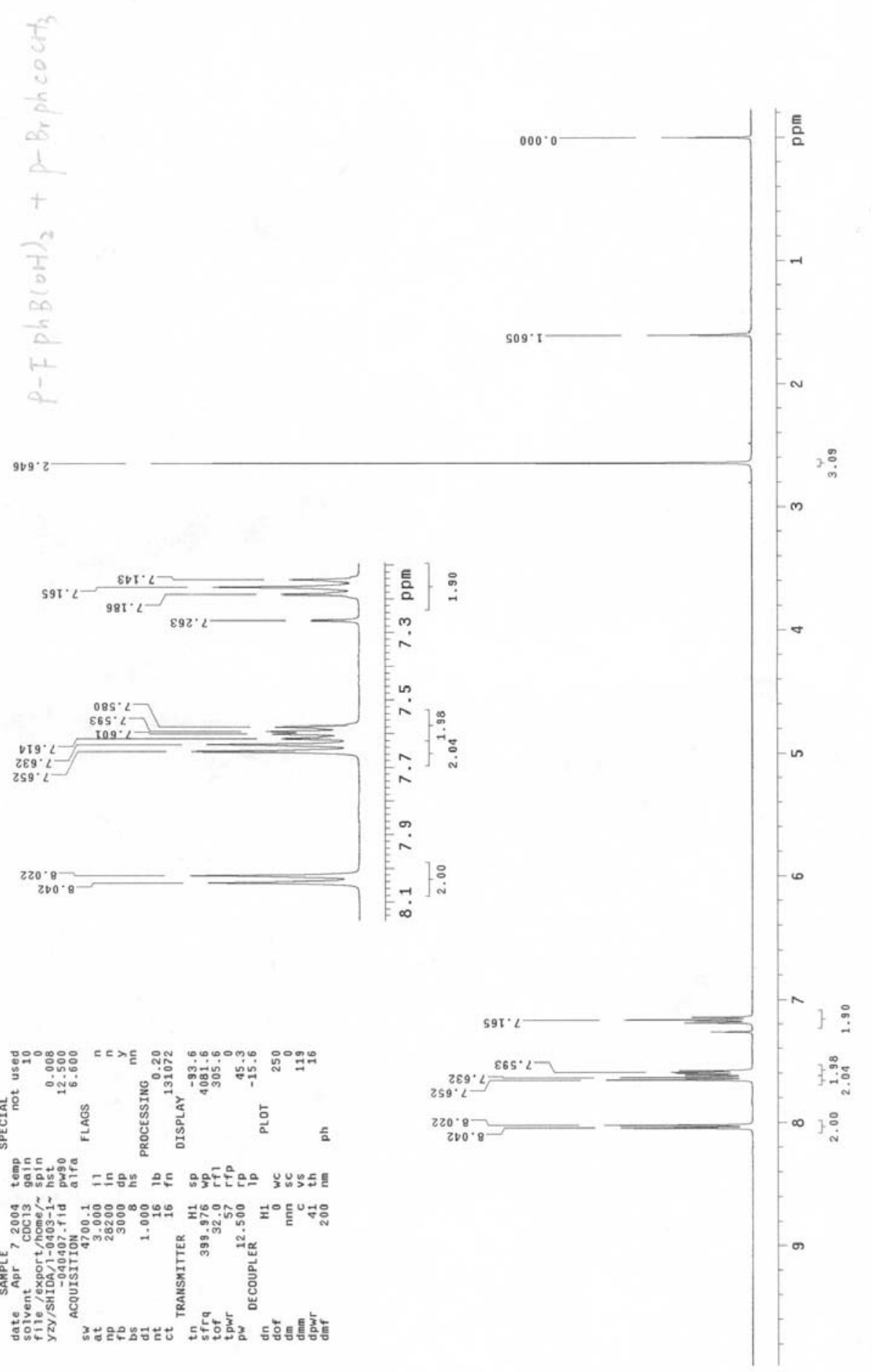


1-(4'-Fluoro-biphenyl-4-yl)-ethanone (3i) $-\mathrm{COCH}_{3}$

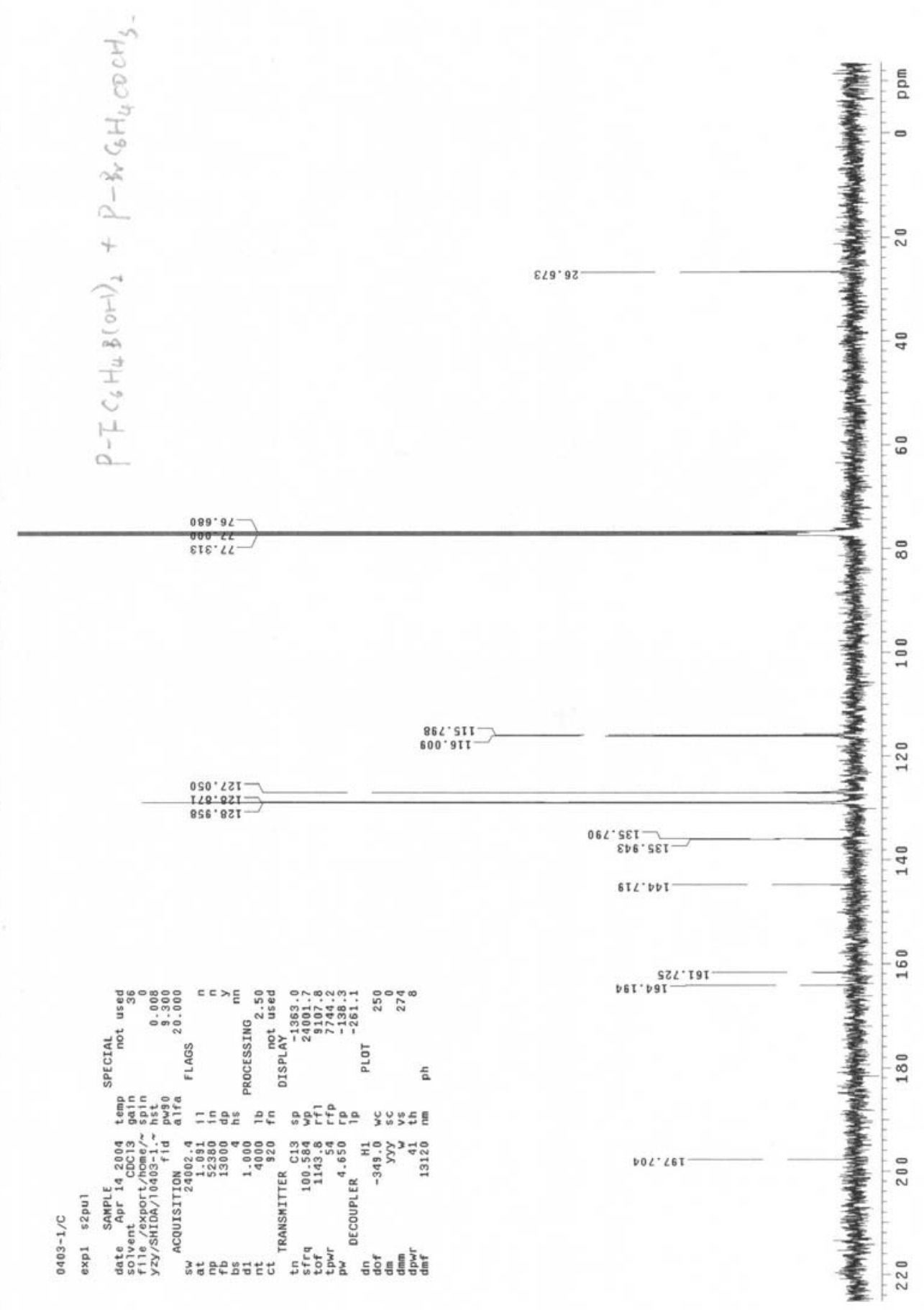


$\left(4,4^{\prime \prime}\right)$-Difluoro-[1,1';4',1"']Terphenyl (3j)

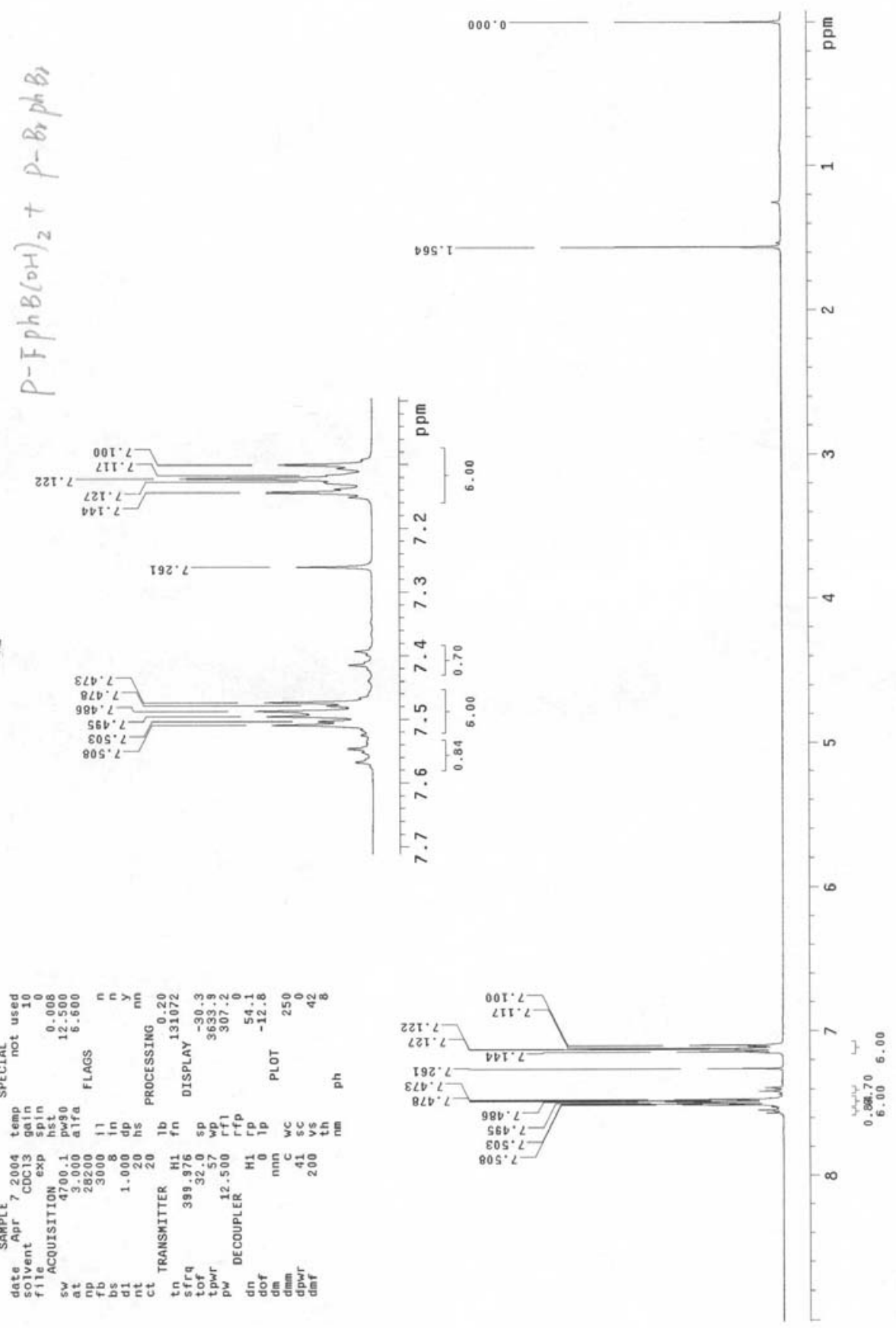


(4, 4')-Difluoro-[1,1';4',1']Terphenyl (3j)
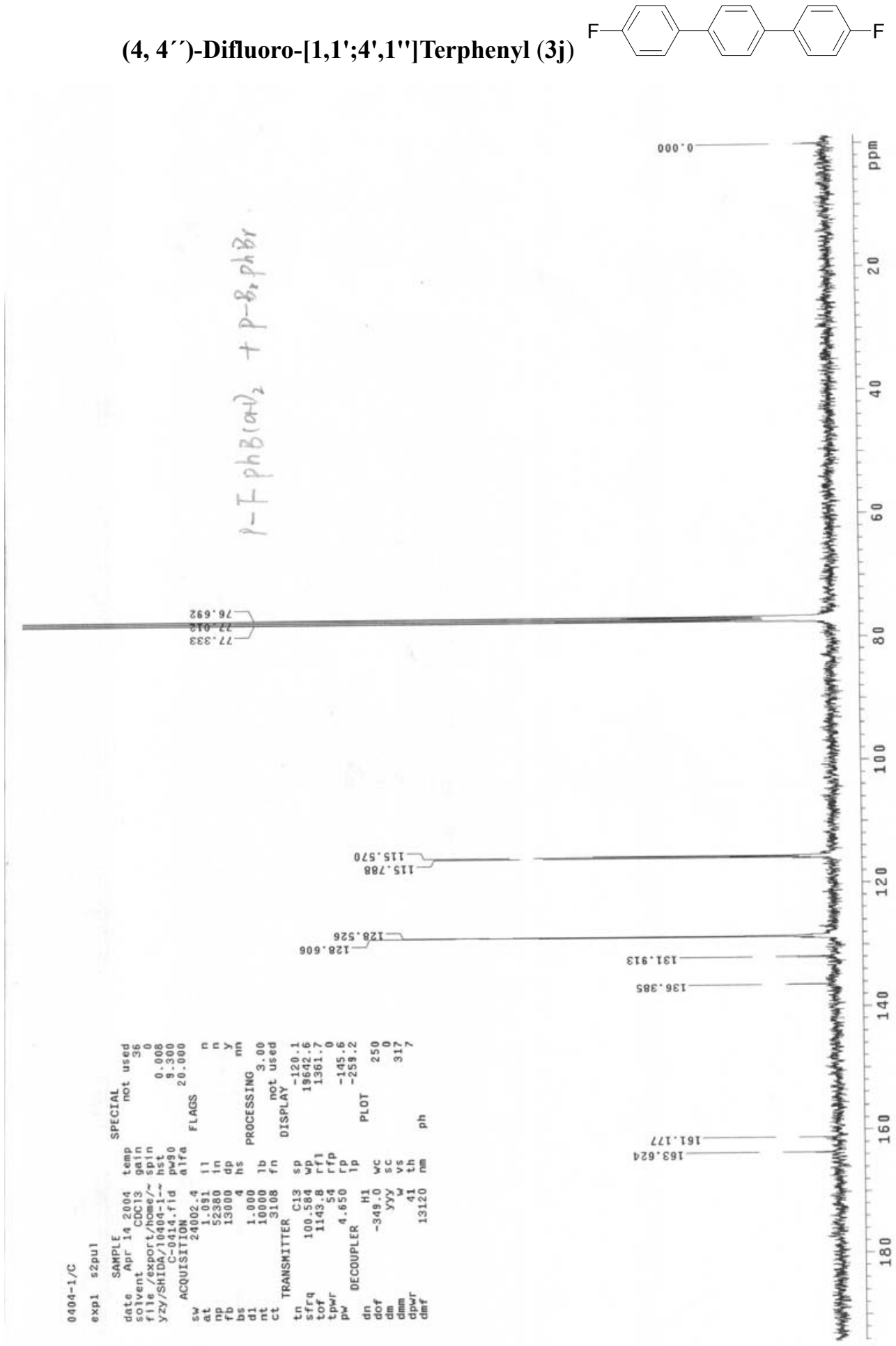